\title{
CORRECTION
}

\section{Correction to: Thoracic and cardiovascular surgery in Japan during 2001}

\author{
Annual report by the Japanese Association for Thoracic Surgery \\ Committee of Science ${ }^{1} \cdot$ Isao Yada $^{2} \cdot$ Hiromi Wada $^{3} \cdot$ Masayuki Shinoda $^{4} \cdot$ Keishu Yasuda $^{5}$ \\ Published online: 15 June 2018 \\ (C) The Japanese Association for Thoracic Surgery 2018
}

\section{Correction to:}

General Thoracic and Cardiovascular Surgery 2001 https://doi.org/10.1007/s11748-003-0016-5

The original publication of the article cited above included incorrect values in the following parts: in Table 3 of section (A) Cardiovascular Surgery, in Table 10 of section (B) General Thoracic Surgery and in Table 1 of section (C) Esophageal Surgery.

The corrected versions are shown in the following page.

The original article can be found online at https://doi.org/10.1007/ s11748-003-0016-5.

Committee of Science

survey-adm@umin.net

1 Tokyo, Japan

2 Department of Thoracic \& Cardiovascular Surgery, Mie University School of Medicine, Mie, Japan

3 Department of Thoracic Surgery, Faculty of Medicine, Kyoto University, Kyoto, Japan

4 Department of Thoracic Surgery, Aichi Cancer Center, Aichi, Japan

5 Department of Cardiovascular Surgery, Hokkaido University School of Medicine, Hokkaido, Japan 


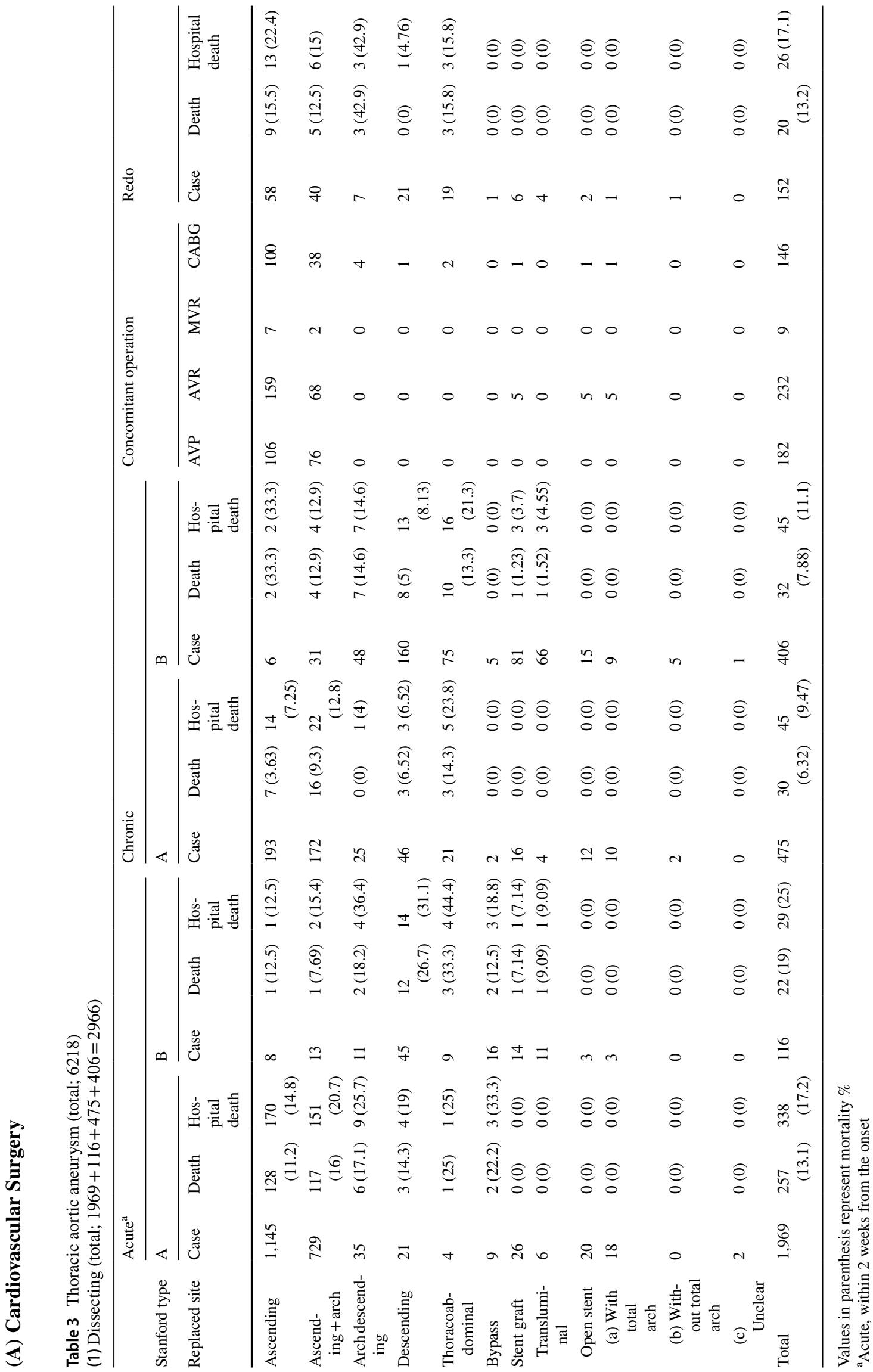


General Thoracic and Cardiovascular Surgery (2018) 66:495-498

497

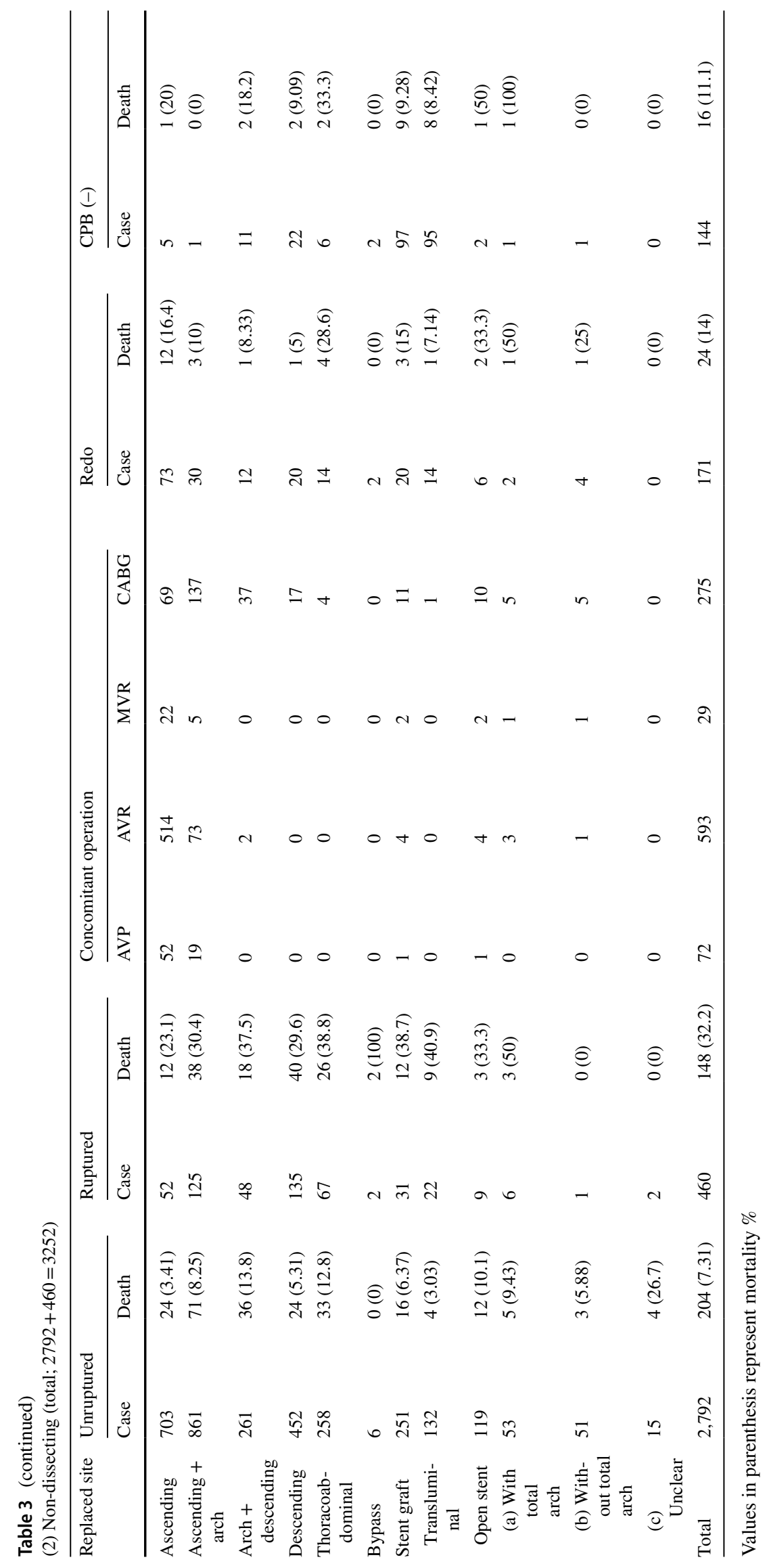

Springer 
(B) General Thoracic Surgery

Table 10

\begin{tabular}{lrllllr}
\hline & $n$ & 30 day death & $\%$ & $\begin{array}{l}\text { Hospital } \\
\text { death }\end{array}$ & HM\% & By VATS \\
\hline $\begin{array}{l}\text { 9. Inflammatory pulmo- } \\
\text { nary disease }\end{array}$ & 2463 & 1 & 0.04 & 6 & 0.24 & 1373 \\
$\begin{array}{l}\text { Tuberculous infection } \\
\text { Fungal infection }\end{array}$ & 789 & 0 & 0.00 & 0 & 0.00 & 449 \\
Bronchiectasis & 323 & 0 & 0.00 & 2 & 0.62 & 104 \\
Others & 90 & 0 & 0.00 & 0 & 0.00 & 19 \\
\hline
\end{tabular}

\section{(C) Esophageal Surgery}

Table 1 Benign esophageal diseases

\begin{tabular}{|c|c|c|c|c|c|c|c|}
\hline & \multicolumn{6}{|c|}{ Operation $(+)$} & \multirow[t]{3}{*}{ Operation (-) } \\
\hline & \multirow[t]{2}{*}{ Total cases } & \multirow[t]{2}{*}{ (Endosc. Surg) } & \multicolumn{4}{|l|}{30 day death } & \\
\hline & & & Total cases & Endosc. Surg & Total cases & Endosc. Surg & \\
\hline 1. Achalasia & 143 & 76 & 1 & 0 & 0 & 0 & 61 \\
\hline 2. Benign tumor & 113 & 49 & 2 & 0 & 1 & 0 & 114 \\
\hline (1) Leiomyoma & 96 & 44 & 2 & 0 & 1 & 0 & 52 \\
\hline (2) Cyst & 8 & 4 & 0 & 0 & 0 & 0 & 13 \\
\hline (3) Others & 10 & 2 & 0 & 0 & 0 & 0 & 13 \\
\hline 3. Diverticulum & 19 & 5 & 1 & 0 & 1 & 0 & 68 \\
\hline 4. Haital hernia & 324 & 134 & 4 & 0 & 3 & 0 & 875 \\
\hline 5. Spontaneous rupture of the esophagus & 54 & 2 & 2 & 0 & 4 & 0 & 180 \\
\hline 6. Esophageal perforation & 31 & 1 & 4 & 0 & 5 & 0 & 35 \\
\hline 7. Esophago-tracheal fistula & 14 & 1 & 0 & 0 & 4 & 0 & 279 \\
\hline 8. Congenital esophageal atresia & 54 & 1 & 3 & 0 & 1 & 0 & 20 \\
\hline 9. Congenital esophageal stenosis & 24 & 10 & 0 & 0 & 0 & 0 & 16 \\
\hline 10. Corrosive stricture of the esophagus & 18 & 1 & 0 & 0 & 0 & 0 & 17 \\
\hline 11. Esophagitis, Esophageal ulcer & 40 & 9 & 0 & 0 & 0 & 0 & 1286 \\
\hline 12. Esophageal varices & 185 & 57 & 4 & 2 & 7 & 2 & 1446 \\
\hline (1) Laparotomy & 132 & 25 & 4 & 2 & 7 & 0 & \\
\hline (2) Sclerotherapy & & & & & & & 1412 \\
\hline 13 Others & 17 & 4 & 1 & 0 & 0 & 0 & 23 \\
\hline Total & 1282 & 425 & 28 & 4 & 34 & 2 & 5910 \\
\hline
\end{tabular}

